\title{
Iron-oxide nanoparticles as a contrast agent in thermoacoustic tomography
}

Xing Jin, Aaron Keho, Kenith Meissner, Lihong V. Wang

Xing Jin, Aaron Keho, Kenith Meissner, Lihong V. Wang, "Iron-oxide nanoparticles as a contrast agent in thermoacoustic tomography," Proc. SPIE 6437, Photons Plus Ultrasound: Imaging and Sensing 2007: The Eighth Conference on Biomedical Thermoacoustics, Optoacoustics, and Acoustooptics, 64370E (13 February 2007); doi: 10.1117/12.698329

SPIE. Event: SPIE BiOS, 2007, San Jose, California, United States 


\title{
Iron oxide nanoparticles as a contrast agent in thermoacoustic tomography
}

\author{
Xing Jin ${ }^{\mathrm{a}}$, Aaron Keho ${ }^{\mathrm{a}}$, Kenith Meissner ${ }^{*}{ }^{\mathrm{a}}$ and, Lihong V. Wang ${ }^{\mathrm{b}}$ \\ ${ }^{a}$ Dept. of Biomedical Engr., Texas A\&M Univ., 3120 TAMU, College Station, TX 77843-3120 \\ ${ }^{b}$ Dept. of Biomedical Engr., One Brooking Dr., Washington Univ. in St. Louis, Campus box 1097, \\ St. Louis, MO 63130-4899
}

\begin{abstract}
We investigate the feasibility of using iron oxide nanoparticles as a contrast agent for radiofrequency (RF) induced thermoacoustic tomography. Aqueous colloids of iron oxide $\left(\mathrm{Fe}_{3} \mathrm{O}_{4}\right)$ nanoparticles have been synthesized and characterized. The synthesis method yielded citrate-stabilized, spherical particles with a diameter of approximately 10 $\mathrm{nm}$. The complex permittivity of the colloids was measured with a coaxial probe and vector network analyzer, and the microwave absorption properties were calculated by using a relationship between the complex permittivity and absorption coefficients. Using our pulsed thermoacoustic imaging system at $3 \mathrm{GHz}$, the time-resolved thermoacoustic responses of those colloids were measured and compared to that of deionized water. Finally, two-dimensional thermoacoustic images were acquired from iron oxide colloids in a tissue phantom. The iron oxide colloids produced an enhancement in RF absorption of up to three times that of deionized water at $3 \mathrm{GHz}$. The enhancement increased rapidly with decreasing frequency of the RF excitation source. A corresponding increase in time-resolved thermoacoustic signal of more than two times was demonstrated. Our results indicate that iron oxide nanoparticles have the potential to produce enhanced thermoacoustic signals and to provide molecular imaging with functionalized contrast agents for thermoacoustic tomography.
\end{abstract}

Keywords: Thermoacoustic tomography (TAT), contrast agent, iron oxide nanoparticle.

\section{INTRODUCTION}

Microwave-induced thermoacoustic tomography (TAT) is a hybrid imaging technique that combines the contrast provided by microwave energy with the spatial resolution of thermoacoustic waves [1][2]. This technique typically uses the endogenous contrast provided primarily by water content and ionic concentration in tissue. While powerful, thermoacoustic tomography would benefit greatly from the development of exogenous contrast agents. Exogenous contrast agents would decouple contrast from penetration depth and open applications in which the endogenous contrast is insufficient, especially in molecular imaging. Iron oxide nanoparticles are chosen because they have resonance in the microwave region. Currently they have been used as exogenous contrast agents in MRI imaging and possess high biocompatibility. In this paper, we investigated the possibility of using citrate-stabilized iron oxide nanoparticles as a contrast agent in TAT. Aqueous colloids of iron oxide $\left(\mathrm{Fe}_{3} \mathrm{O}_{4}\right)$ nanoparticles were prepared in our lab with a diameter of approximately $10 \mathrm{~nm}$ and surface stabilized by citrate. We measured the complex permittivity of the colloids with a coaxial dip probe and a vector network analyzer, and then calculated the microwave absorption rate based on a relationship between the dielectric properties and absorption coefficients of a test sample. Next, the time-resolved thermoacoustic responses of the colloids were measured and compared to that of water using our $3 \mathrm{GHz}$ TAT system.

* To whom all correspondence should be addressed. Telephone: 979-458-0180; Fax: 979-845-4450;

Email: kmeissner@tamu.edu

Photons Plus Ultrasound: Imaging and Sensing 2007: The Eighth Conference on Biomedical Thermoacoustics, Optoacoustics, and Acousto-optics, edited by Alexander A. Oraevsky, Lihong V. Wang,

Proc. of SPIE Vol. 6437, 64370E, (2007) - 1605-7422/07/\$18 - doi: 10.1117/12.698329

Proc. of SPIE Vol. 6437 64370E-1 
Finally, two-dimensional thermoacoustic signals were acquired from iron oxide colloids in a tissue phantom, and then TAT images were reconstructed by using a filtered backprojection method.

\section{METHODS}

\subsection{Energy deposition in TAT}

The propagation of an electromagnetic field in a medium is defined by the relationship $E=E_{0} e^{j \omega t-\gamma(\omega) z}$, where $E$ is the electric field at a distance $Z$ from the source of field strength $E_{0}$. The propagation constant $\gamma(\omega)$ can be written as $\gamma(\omega)=\alpha(\omega)+j \beta(\omega)$, where $\alpha(\omega)$ and $\beta(\omega)$ are the attenuation constant and phase constant at angular frequency $\omega$, respectively. The energy deposition by the electromagnetic radiation at a specific point in tissue is determined by its dielectric properties and the electrical field at that specific location. To find the total rate of energy absorbed by an object, we use specific absorption rate (SAR), which is defined as $S A R=\sigma|E|^{2} / \rho$, where $\rho$ is the mass density of the object at that point and $\sigma$ is the conductivity of the tissue [3]. We will show that attenuation constant can be derived from the dielectric properties of a material in the next section. Therefore, if we know the dilectric properties of a tissue, we can estimate the strength of the electric field and the conductivity of the tissue at a specific point, and then we can easily estimate the energy deposition at that location.

To evaluate the effectiveness of a contrast agent for TAT, we need to estimate the electrical field within the tissue. In our experiments the sizes of test samples are small compared with the wavelength of microwave. Thus, we assume uniform electric-field distribution in test samples to simplify the computation. Also as shown in the definition of SAR, the phase constant of the electric field does not have any effects on the value of energy deposition, therefore we only need to find attenuation constant of the tissue. Moreover, because the attenuation of an electromagnetic field in a medium is mainly brought by dielectric loss, we will use $\alpha(\omega)$ to evaluate the microwave absorption coefficient.

\subsection{Computation of absorption coefficient}

Permittivity relates to a material's ability to transmit an electric field. The complex relative permittivity $\varepsilon^{*}(\omega)$ is defined as $\varepsilon^{*}(\omega)=\varepsilon_{r}(\omega)-j \varepsilon_{i}(\omega)$, where $\varepsilon_{r}$ and $\varepsilon_{i}$ are the real and imaginary parts of the complex relative permittivity respectively. We will use two different definitions of the complex refraction index to derive the absorption coefficient of a material. The complex refraction index $n^{*}(\omega)$ for a given material is defined as the square root of the product of the complex relative permittivity and complex relative permeability,

$$
n^{*}(\omega)=\sqrt{\varepsilon^{*}(\omega) \mu^{*}(\omega)}
$$

The complex refraction index can also be derived from the extinction coefficient of tissue and is given by

$$
n^{*}(\omega)=n(\omega)-j k(\omega)
$$

where $k(\omega)$ is the extinction coefficient. And we also have $k(\omega)=\lambda \alpha(\omega) /(2 \pi)$. From the above relationships, through some simple derivations, we can obtain the microwave absorption coefficient of a material as:

$$
\alpha(\omega)=\frac{\omega}{c_{0}} \sqrt{\frac{\mu_{r}(\omega) \varepsilon_{r}(\omega)}{2}\left(\sqrt{1+\left(\frac{\sigma(\omega)}{\omega \varepsilon_{0} \varepsilon_{r}(\omega)}\right)^{2}}-1\right)}
$$


where $\mu_{r}(\omega)$ is real component of the relative complex permeability at angular frequency $\omega, c_{0}$ is the speed of electromagnetic waves in vacuum (approximately $3 \times 10^{8} \mathrm{~m} / \mathrm{s}$ ), and $\sigma(\omega)$ is the conductivity which is defined as $\omega \varepsilon_{0} \varepsilon_{i}(\omega) \quad\left(\varepsilon_{0}=8.85 \times 10^{-12} \mathrm{~F} / \mathrm{m}\right)$. $1 / e$ penetration depth $\mu$ in the tissue is defined as $1 / \alpha(\omega)$. In our thermoacoustic tomography system, the electric field is much stronger than the magnetic field. We will use Eq. (3) to calculate microwave absorption coefficients in the following experiments. When the magnetic properties can not be ignored, we, however, need to measure both the permittivity and permeability of the material to get a reasonable estimate of the absorption coefficient. Kim et. al. [4] provides a simple experimental method to determine the microwave energy deposition for this case.

\subsection{Measurement of absorption coefficients}

Among the different ways to measure relative permittivity, a coaxial probe is ideal for liquids and semi-solid materials [5][6], and has thus been chosen for our application. The open-ended coaxial probe can be regarded as a cut-off section of a transmission line. The dielectric properties of a material are measured by immersing the probe in a liquid or touching it to the flat face of a semi-solid test sample. The electric fields at the probe tip propagate into the test sample and vary as they come into contact with different test samples. The reflected signals from the test sample are then measured and related to the complex relative permittivity.

The main measurement system includes a coaxial probe kit, a network analyzer and a RF source. The microwave absorption coefficients are calculated using Eq. (3). The network analyzer measures the complex relative permittivity. The schematic graph of the experimental setup is shown in Fig. 1. We use an Agilent 8510C vector network analyzer to make broadband measurements from $200 \mathrm{MHz}$ to $20 \mathrm{GHz}$. We use the Agilent 85070E dielectric probe kit which includes a coaxial probe and the corresponding software. An external computer controls the network analyzer through GPIB. Before each measurement, a calibration at the tip of the probe must be performed. The principle of the calibration is to use the difference between the predicted and actual values of three well-known standards (air, a short circuit and deionized water) to remove the repeatable systematic errors from the measurement. Before making a measurement, we performed a system calibration. During the experiments, it is important to make sure that the cable is stabilized and not flexed between the calibration and measurement. The air bubbles on the tip of the probe need to be carefully removed to ensure the accuracy of a measurement. And the test sample must also be thick enough to appear infinite to the probe.

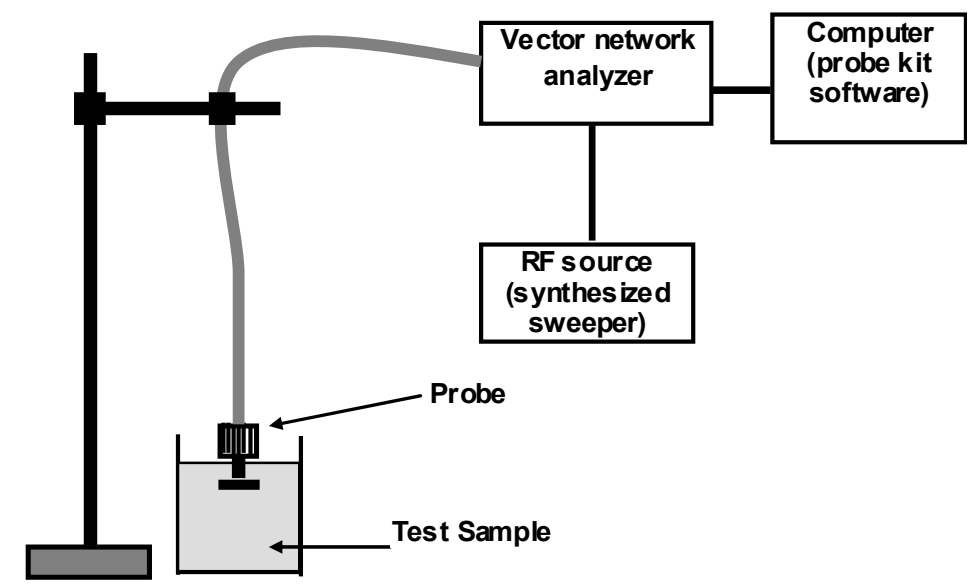

Fig. 1 Schematic graph of the experimental setup for measuring dielectric properties 


\subsection{TAT experiment}

A schematic experimental setup for TAT has been shown in Fig. 2. The central frequency of the microwave source is 3 GHz. The pulse width of the microwave source is $0.5 \mu \mathrm{s}$. The peak power of the microwave pulse is estimated to be 2 $\mathrm{kW}$, and the estimated total energy of the microwave pulse is about $1 \mathrm{~mJ}$. The microwave radiation level of this TAT system is estimated to be below the safety requirement [3]. The tissue absorbs the pulsed microwave energy, and generates thermoacoustic waves below $2 \mathrm{MHz}$. We therefore use an unfocused transducer (V323, Panametrics Inc.) with a central frequency of $1 \mathrm{MHz}$ as the receiver. The transducer is mounted on a mechanical arm. The position of the transducer is fixed when measuring the time-resolved thermoacoustic signal. To obtain two dimensional TAT image, the ultrasonic transducer, controlled by a stepping motor, scans the tissue sample circularly to acquire two dimensional projection data.

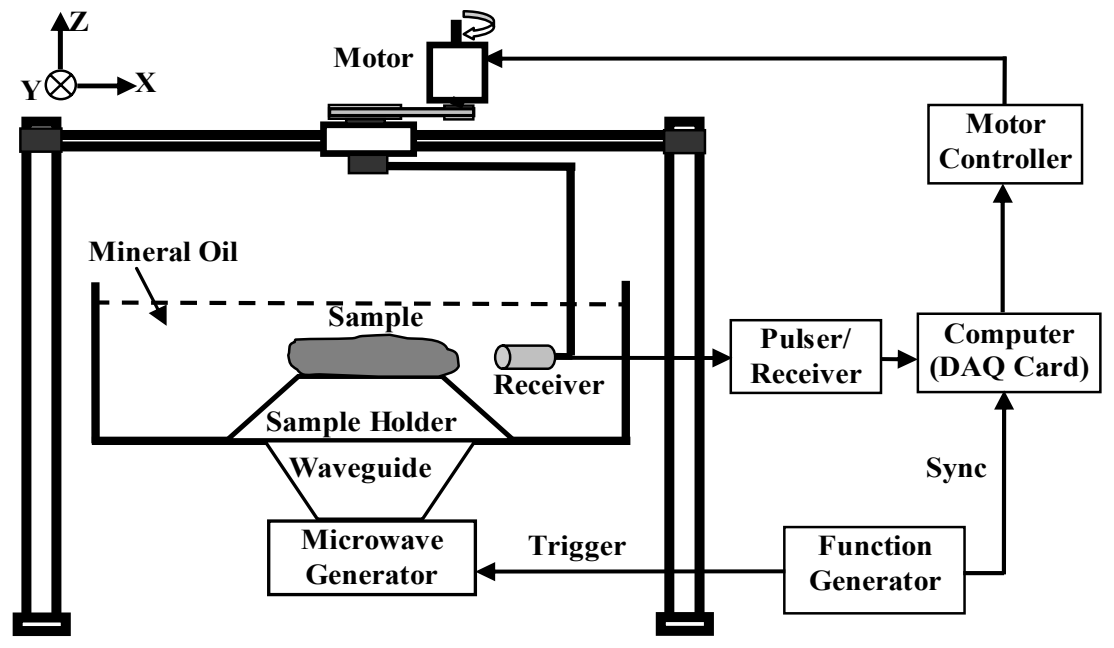

Fig. 2 Schematic graph of the TAT setup

\section{RESULTS}

\subsection{Absorption coefficients}

An aqueous suspension of $\mathrm{Fe}_{3} \mathrm{O}_{4}$ nanoparticles was prepared with diameters ranging 8-10nm with the surfaces functionalized by citrate. After the colloid was stabilized, we separated the supernatant as the test sample, and then diluted the supernatant at 2,5 and 10 times with deionized water. Because water content in the tissue is the main contributor to microwave absorption, we used deionized water as the control sample in our experiments. The dielectric properties of test samples were then measured by using the method discussed in section 2.3. The results are shown in the Fig. 3. Figure 3(a) shows the real component of complex relative permittivity, and Fig. 3(b) shows the imaginary component of complex relative permittivity. The supernatant is weaker in $\varepsilon_{r}$ and stronger in $\varepsilon_{i}$ as compared to that of deionized water. We know that the imaginary component of the complex relative permittivity $\varepsilon_{i}$ is related to the conductivity of a material. Because fig. 3(b) shows big differences in $\varepsilon_{i}$ of different test samples, we expect significant changes in their microwave absorption coefficients. In Fig. 3(c), we find that the microwave absorption rate of the supernatant is approximately three times stronger than that of deionized water at $3 \mathrm{GHz}$. If we dilute the supernatant, then the microwave absorption rate of the diluted colloid drops accordingly. 


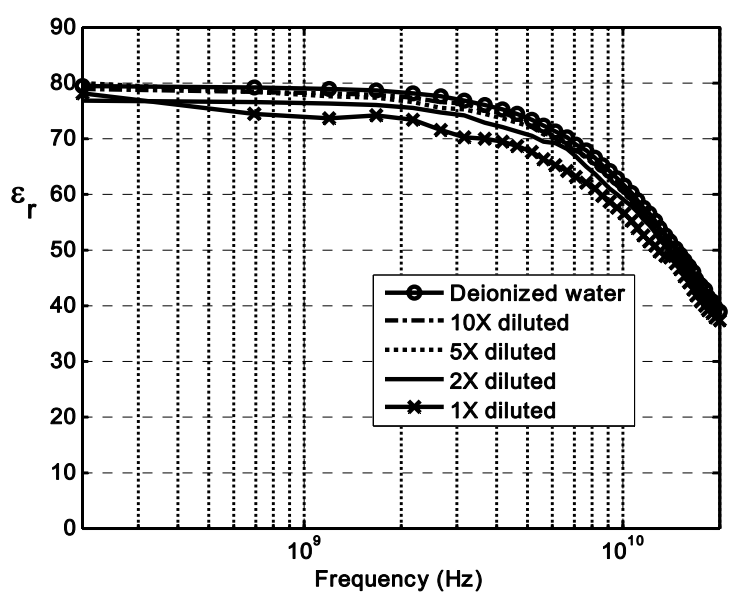

(a)

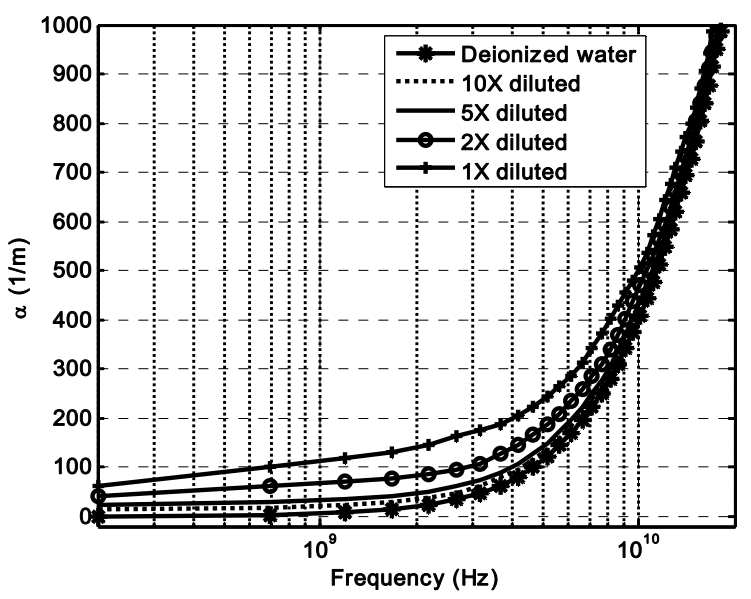

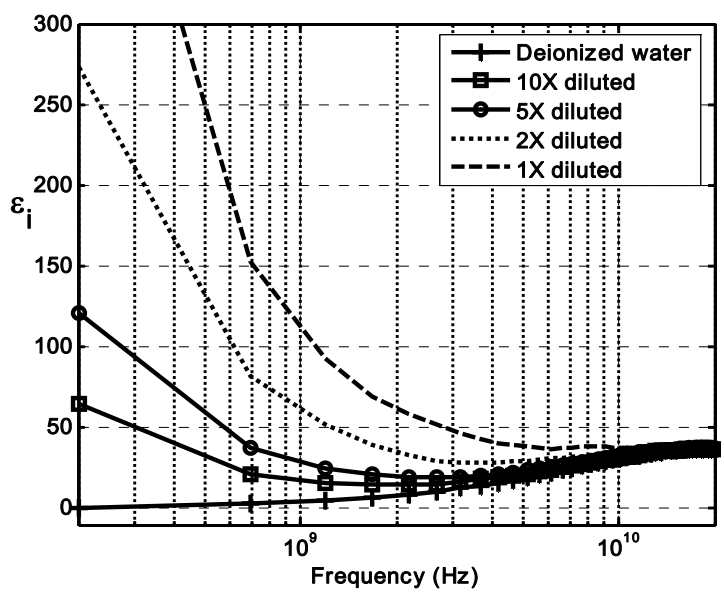

(b)

(c)

Fig. 3 Dielectric properties and absorption coefficients (a) real component of complex relative permittivity of test samples, (b) imaginary component of complex relative permittivity of test samples, (c) calculated microwave absorption coefficients.

\subsection{Comparison of time-resolved thermoacoustic signals}

To compare the time-resolved thermoacoustic signals of the iron oxide colloids, we filled a piece of plastic tubing with colloids containing different concentrations of iron oxide nanoparticles. The inner diameter of the tubing was $1 / 16^{\prime \prime}$. The tube was fixed at two ends above the microwave waveguide. The position of the tube was kept unchanged during all the tests. We began with the colloid at the lowest concentration of iron oxide nanoparticles and then gradually increased the concentration of iron oxide nanoparticles. In each experiment, we carefully cleaned the tubing before filling a new test sample, thus the influence of the remaining colloids from previous measurement on the current measurement was minimized. Using our pulsed thermoacoustic imaging system at $3 \mathrm{GHz}$, the time-resolved thermoacoustic responses of the colloids with different concentrations of iron oxide nanoparticles were measured and compared to that of deionized water in Fig. 4(a). Peak values in the thermoacoustic responses of iron oxide colloids were then normalized to that of deionized water and plotted in Fig. 4(b). The strength of thermoacoustic signal generated by iron oxide colloids was more than two times that of deionized water at the highest concentration. The higher the concentration of iron oxide nanoparticles in the colloids was, the stronger the strength of time-resolved thermoacoustic signals was. 


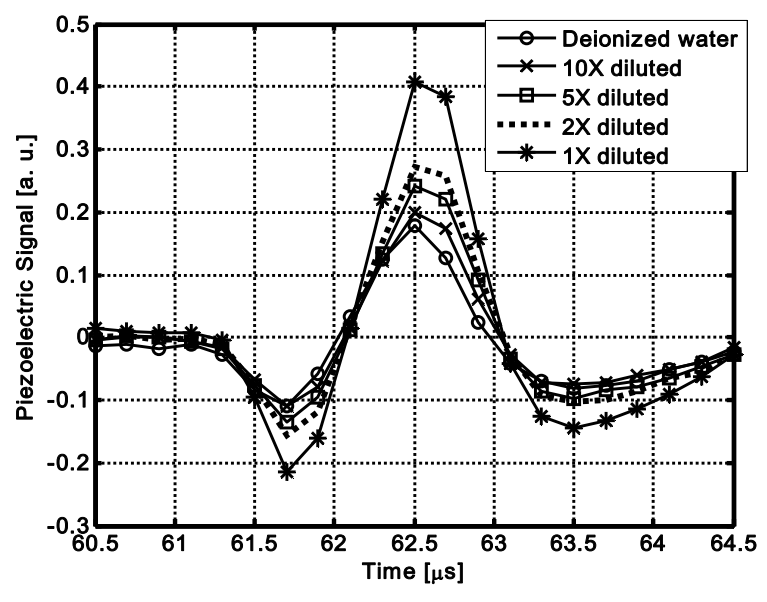

(a)

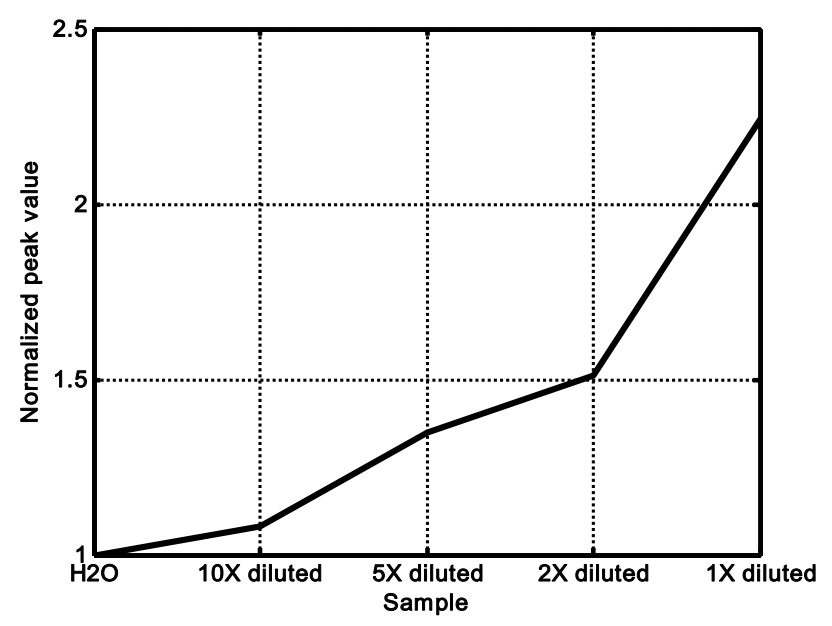

(b)

Fig. 4 Time-resolved thermoacoustic signal (a) Comparisons of time-resolved thermoacoustic signals generated by test samples and (b) line plot of the normalized peak values in (a).

\subsection{Two-dimensional TAT image}

We made a tissue phantom by filling two small tubes with deionized water and two small tubes with the 1x diluted sample discussed in section 3.1. All the four tubes were fixed in a piece of porcine fat and sealed on the top and bottom. We choose porcine fat because it has a low microwave absorption rate and thus will not interfere with the results. The whole phantom was then immersed in mineral oil and placed on a sample base on the X-Y plane. The data collection process has been detailed in section 2.4. The image was reconstructed by using a filtered backprojection method [7]. Figure 5(a) is the reconstructed TAT image. Two tubes filled with supernatant sample are shown as bright spots marked by two white arrows, and two tubes filled with deionized water are denoted by two black arrows. In Fig. 5(b), we compared the signal strength at a depth marked by two black dashed arrows in Fig 5(a). Our results showed an increased contrast of approximately three times at $3 \mathrm{GHz}$ by using the contrast agent.

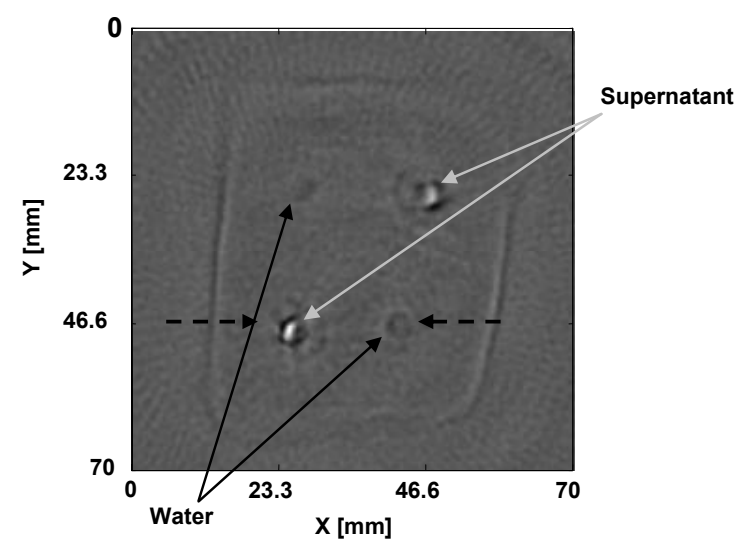

(a)

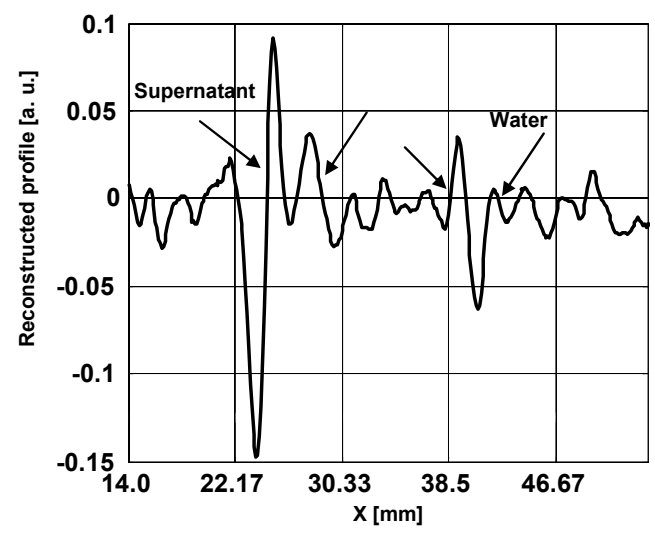

(b)

Fig. 5 TAT image (a) Two tubes filled with supernatant sample show as the bright spots in the image, and two tubes filled with deionized water show as the dark circles in the image, (b) Line profile at the depth marked by two black dashed arrows in fig. 5(a). 


\section{CONCLUSIONS}

We presented our preliminary results using iron oxide nanoparticles as a contrast agent in thermoacoustic tomography. It had been shown that the contrast agent colloids can produce an increase in microwave absorption of up to three times that of deionized water at $3 \mathrm{GHz}$. Our experimental results also demonstrated a corresponding increase in time-resolved thermoacoustic signal of more than two times. The contrast agent colloids also showed an increased contrast of approximately three times in two-dimensional TAT image. Our results indicate that iron oxide nanoparticles have the potential to expand applications of thermoacoustic tomography in molecular imaging and diagnostic imaging.

\section{ACKNOWLEMENTS}

We would like to thank Shih-hsun Hsu, Rui Xu, and Dr. Kai Chang for their assistance with microwave measurement. This project was sponsored by National Institutes of Health grants R01 EB000712 and R01 NS46214.

\section{REFERENCES}

1. L.-H. Wang, X. Zhao, H. Sun, and G. Ku, "Microwave-induced acoustic imaging of biological tissues," Rev. Sci. Instr., vol. 70, 3744-3748 (1999)

2. R. A. Kruger, K. M. Stantz, W. L. Kiser Jr., "Thermoacoustic CT of the Breast," Proc. SPIE, 4682, 521-525 (2002)

3. P. Stavroulakis ed., Biological Effects of Electromagnetic Fields: Mechanisms, Modeling, Biologica Effects, Therapeutic Effects, International Standards, Exposure Criteria, Springer (2003)

4. D. K. Kim, M. S. Amin, S. Elborai, S.-H. Lee, Y. Koseoglu, M. Zahn and M. Muhammed, "Energy absorption of superparamagnetic iron oxide nanoparticles by microwave irradiation", Jour. Appl. Phys., 97 (2005)

5. S. Ray and J. Behari, "Microwave absorption in lossy liquids", Phys. Med. Biol., vol. 31, no. 9, 1031-1040 (1986)

6. D. V. Blackham, R. D. Pollard, "An Improved Technique for Permittivity Measurements Using a Coaxial Probe." IEEE Trans. Instr. Meas., vol. 46, no. 5, 1093- 1099 (1997)

7. Y. Xu, P. Kuchment, and L.-H. Wang, "Limited-view thermoacoustic tomography and reconstruction by truncated conjugate gradient," Med. Phys. vol. 31, 724-733 (2004) 